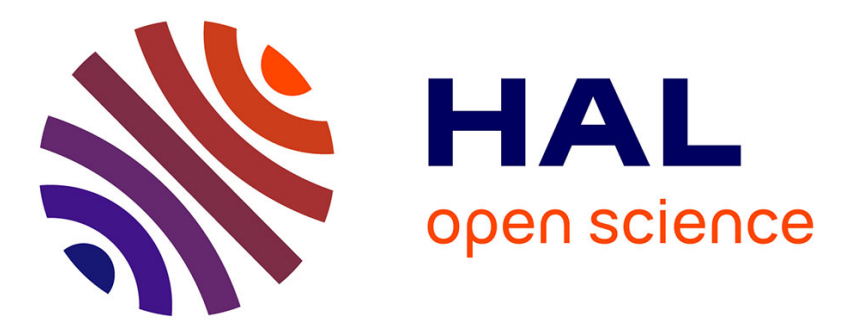

\title{
Laguerre-Gaussian quasi-modal q-plates from nanostructured glasses
}

Mushegh Rafayelyan, Titas Gertus, Etienne Brasselet

\section{To cite this version:}

Mushegh Rafayelyan, Titas Gertus, Etienne Brasselet. Laguerre-Gaussian quasi-modal q-plates from nanostructured glasses. Applied Physics Letters, 2017, 110 (26), pp.261108. 10.1063/1.4990954 . hal-01565752

\section{HAL Id: hal-01565752 \\ https://hal.science/hal-01565752}

Submitted on $20 \mathrm{Jul} 2017$

HAL is a multi-disciplinary open access archive for the deposit and dissemination of scientific research documents, whether they are published or not. The documents may come from teaching and research institutions in France or abroad, or from public or private research centers.
L'archive ouverte pluridisciplinaire HAL, est destinée au dépôt et à la diffusion de documents scientifiques de niveau recherche, publiés ou non, émanant des établissements d'enseignement et de recherche français ou étrangers, des laboratoires publics ou privés.

\section{다(1)(2)}

Distributed under a Creative Commons Attribution - ShareAlikel 4.0 International 


\section{Laguerre-Gaussian quasi-modal q-plates from nanostructured glasses}

Mushegh Rafayelyan, Titas Gertus, and Etienne Brasselet

Citation: Appl. Phys. Lett. 110, 261108 (2017); doi: 10.1063/1.4990954

View online: http://dx.doi.org/10.1063/1.4990954

View Table of Contents: http://aip.scitation.org/toc/apl/110/26

Published by the American Institute of Physics

\section{Articles you may be interested in}

As-grown two-dimensional $\mathrm{MoS}_{2}$ based photodetectors with naturally formed contacts

Applied Physics Letters 110, 261109 (2017); 10.1063/1.4990968

Extraordinary optical transmission through nonlocal holey metal films

Applied Physics Letters 110, 261110 (2017); 10.1063/1.4991016

Electrical switching of the magnetic vortex circulation in artificial multiferroic structure of $\mathrm{Co} / \mathrm{Cu} / \mathrm{PMN}-\mathrm{PT}(011)$

Applied Physics Letters 110, 262405 (2017); 10.1063/1.4990987

Electrode modulated capacitance-electric field nonlinearity in metal-insulator-metal capacitors

Applied Physics Letters 110, 263503 (2017); 10.1063/1.4989531

A highly efficient element for generating elliptic perfect optical vortices

Applied Physics Letters 110, 261102 (2017); 10.1063/1.4990394

Directly generating orbital angular momentum in second-harmonic waves with a spirally poled nonlinear photonic crystal

Applied Physics Letters 110, 261104 (2017); 10.1063/1.4990527

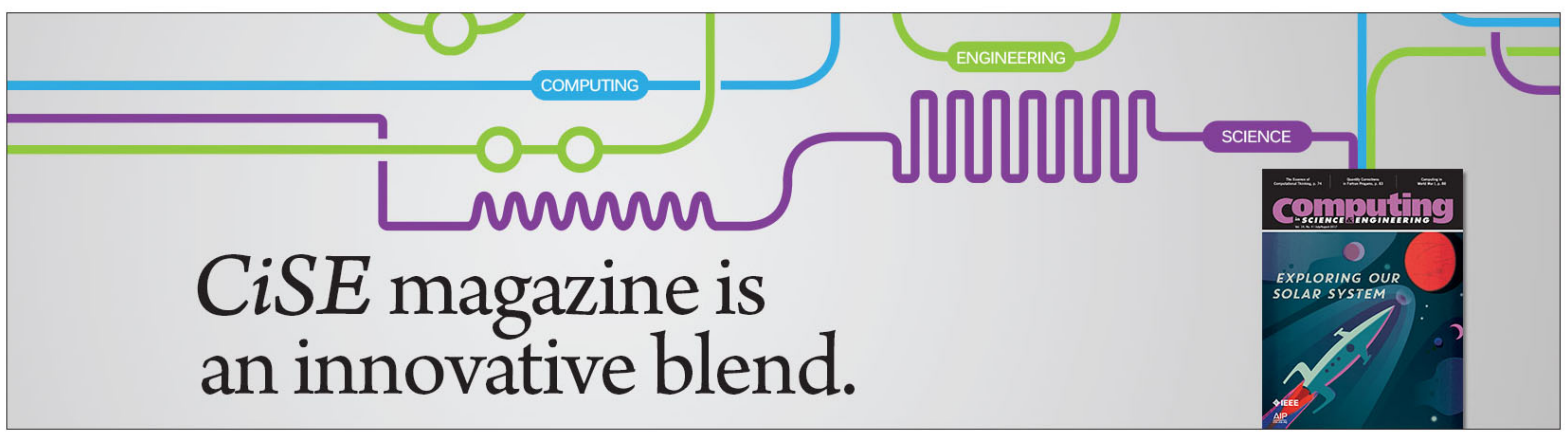




\title{
Laguerre-Gaussian quasi-modal q-plates from nanostructured glasses
}

\author{
Mushegh Rafayelyan, ${ }^{1}$ Titas Gertus, ${ }^{2}$ and Etienne Brasselet ${ }^{1, a)}$ \\ ${ }^{1}$ University Bordeaux, CNRS, LOMA, UMR 5798, F 33400 Talence, France \\ ${ }^{2}$ Altechna R\&D Ltd., Mokslininku St. 6A, Vilnius LT 08412, Lithuania
}

\begin{abstract}
A quasi-modal version of the recently introduced Laguerre-Gaussian modal q-plates [Rafayelyan and Brasselet, Opt. Lett. 42, 19661969 (2017)] is proposed and implemented using femtosecond direct laser writing of space-variant nanogratings in the bulk of silica glass. The corresponding design consists of linear azimuthal modulation of the optical axis orientation and polynomial radial modulation of the retardance profile. Experimental demonstration is made for Laguerre-Gaussian modes with azimuthal indices $l=(1,2,3)$ and radial index $p=0$. Such quasi-modal q-plates overcome previous limitations regarding the robustness of modality against the handedness of the incident circular polarization state.
\end{abstract}

In optics, Laguerre-Gaussian (LG) modes are exact solutions of the scalar paraxial Helmholtz equation in cylindrical coordinate system $(r, \phi, z){ }^{1}$ They are widely used in the study of light fields carrying orbital angular momentum since the pioneering work by Allen and coworkers who recognized that $L G$ beams with azimuthal index $l \in \mathbb{Z}$ carry an amount of orbital angular momentum per photon proportional to $l{ }^{2}$ whose jubilee has been celebrated recently in a review paper. ${ }^{3}$ In addition, the radial structure of LG beams is associated with a radial index $p \in \mathbb{N}$ that remarkably attracted much less interest although its fundamental and applied interest nowadays leaves no doubt. From a practical point of view, the realization of optical components enabling the controlled generation of $\mathrm{LG}_{l, p}$ modes is a long-standing issue and we refer to Ref. 4 for a concise overview. Here, we address the experimental implementation of a recent theoretical proposal based on the use of inhomogeneous and anisotropic optical elements. ${ }^{4}$

In Ref. 4, it has been shown that a structural upgrade of a so-called q-plate $(\mathrm{QP}$, that is a slab of uniaxial optical material with uniform birefringent phase retardation $\Delta=\pi$ and azimuthally varying optical axis orientation $\psi=q \phi$ with $q$ half-integer) may lead to the generation of circularly polarized $\mathrm{LG}_{l, p}$ optical modes from circularly polarized Gaussian beams with opposite handedness. This differs from the original QP design that corresponds to a polarizationcontrolled helical pure phase mask ${ }^{5,6}$ whose applied and fundamental interests in classical and quantum optics are broad. ${ }^{7}$ More specifically, the required analytical expressions $\Delta_{l, p}(r)$ and $\psi_{l, p}(r, \phi)$ for the radially modulated anisotropic properties of a QP have been derived, assuming that its extraordinary refractive index can be modulated at a fixed ordinary refractive index. ${ }^{4}$ However, obtained theoretical profiles having $p+1$ cusps along the radial coordinate are associated with discontinuous derivative functions $\partial \Delta_{l, p}(r) /$ $\partial r$ and $\partial \psi_{l, p}(r, \phi) / \partial r$, which do not ease the fabrication of modal QPs. Moreover, as noticed in Ref. 4, the latter design breaks the right/left symmetry and prevents the

a)etienne.brasselet@u bordeaux.fr polarization-controlled transformation $\mathrm{LG}_{l, p} \rightarrow \mathrm{LG}_{l, p}$ by flipping the helicity $(\sigma= \pm 1 \rightarrow \sigma=\mp 1)$ of a normally incident circularly polarized Gaussian beam owing to the relationship $l=2 \sigma q{ }^{6}$

We propose to get rid of above technological challenges and fundamental issues by simplifying the original design in the limit of small birefringent phase retardation. For this purpose, we consider an incident $\sigma$-circularly polarized incident Gaussian beam with waist radius $w_{0, \text { in }}$ located at the plane of the sample characterized by $\Delta(r, \phi)$ and $\psi(r, \phi)$. Following Ref. 4 , the contra-circularly polarized output field emerging from the optical element is

$$
E_{\text {out }}^{(\sigma)}(r, \phi) \propto \sin \left[\frac{\Delta(r, \phi)}{2}\right] e^{\frac{r^{2}}{w_{0, \text { in }}^{2}}+\frac{\Delta(r, \phi)}{2}+i 2 \sigma \psi(r, \phi)} .
$$

Then, in the limit of small retardance, one can show that the following profiles:

$$
\begin{gathered}
\Delta_{l, p}(r)=\Delta_{0}\left(r / w_{0, \text { out }}\right)^{|l|} L_{p}^{|l|}\left(\frac{2 r^{2}}{w_{0, \text { out }}^{2}}\right) e^{\frac{r^{2}}{w_{0, \text { in }}^{2}}} \frac{r^{2}}{w_{0, \text { out }}^{2}}, \\
\psi_{l, p}(\phi)=\sigma l \phi / 2+\psi_{0},
\end{gathered}
$$

where $\Delta_{0}$ and $\psi_{0}$ are constants and $L_{p}^{|l|}(x)=\sum_{k=0}^{p} \frac{(|l|+p) !(x)^{k}}{(|l|+k) !(p k) ! k !}$ are the associated Laguerre polynomials, gives a $\mathrm{LG}_{l, p}$ mode with waist radius $w_{0, \text { out }}$. Remarkably, obtained expressions for the birefringent phase retardation and optical axis orientation angle correspond to differentiable functions, hence smooth structuring patterns. Also, the right/left symmetry is no longer broken. This demonstrates the possibility to realize quasi-modal q-plates (QMQPs) with polarizationcontrolled azimuthal index and polarization-independent modality as long as $\Delta_{0}$ is not too large.

Quantitatively, the modal figure of merit of such QMQP is assessed by evaluating the power fraction $\rho_{l, p}$ of $-\sigma$-circularly polarized field component just at the output of the optical element that corresponds to $\mathrm{LG}_{l, p}$ beam (assuming $\sigma$-circularly polarized incident Gaussian beam). This is done by projecting the contra-circularly polarized output field emerging from the optical element on the $\mathrm{LG}_{l, p}$ mode 


$$
E_{l, p}(r, \phi ; w) \propto\left(\frac{r}{w}\right)^{|l|} L_{p}^{|l|}\left(\frac{2 r^{2}}{w^{2}}\right) e^{\frac{r^{2}}{w^{2}}+i l \phi},
$$

characterized by the waist radius $w$. Namely,

$$
c_{l, p}(w)=\frac{\int_{0}^{\infty} E_{\text {out }}^{(\sigma)} E_{l, p}(w)^{*} r \mathrm{~d} r}{\sqrt{\int_{0}^{\infty}\left|E_{\text {out }}^{(\sigma)}\right|^{2} r \mathrm{~d} r \int_{0}^{\infty}\left|E_{l, p}(w)\right|^{2} r \mathrm{~d} r}},
$$

where asterisk denotes complex conjugation. Note that the dependence of the projection coefficient $c_{l, p}$ on $w$ recalls that the decomposition of a paraxial light field on LG basis is not unique. ${ }^{89}$ Accordingly, the modality is evaluated as

$$
\rho_{l, p}=\max _{w}\left|c_{l, p}(w)\right|^{2} .
$$

Considering the typical case $w_{0, \text { in }}=w_{0, \text { out }}$, we evaluate the dependence of the modality parameter on $\Delta_{0}$. The results are displayed in Fig. 1(a) for $l=(1,2,3)$ and $p=0$. As expected, $\rho_{l, p} \rightarrow 1$ when $\Delta_{0} \rightarrow 0$ and $\rho_{l, p}$ substantially decreases as $\Delta_{0}$ increases. Note that the maximization with respect to $w$ in Eq. (6) gives a moderate monotonous decrease from $w_{0, \text { out }}$ for $\Delta_{0} \rightarrow 0$ down to $w \simeq$ $(0.92,0.86,0.82) \times w_{0, \text { out }}$ for $l=(1,2,3)$ in the range of $\Delta_{0}$ where modal performances are better than usual QPs [see dashed lines in Fig. 1(a)]. We conclude that the proposed design quantitatively improves the modality. Moreover, we notice a drastic qualitative improvement regarding the generation of doughnut-like intensity patterns just after the optical element, hence the self-similar character of the LG beam shaping. This is illustrated in Figs. 1(b) 1(d) where the calculated [using Eq. (1)] targeted LG beam profile [panel (b)] is compared to QMQP with $\Delta_{0}=\pi / 5$ [panel (c)] and QP [panel (d)] for $(l, p)=(1,0)$. Still, in all cases, the field has a helical phase profile around an optical phase singularity of topological charge $l$, as shown in Fig. 1(e). Of course, the
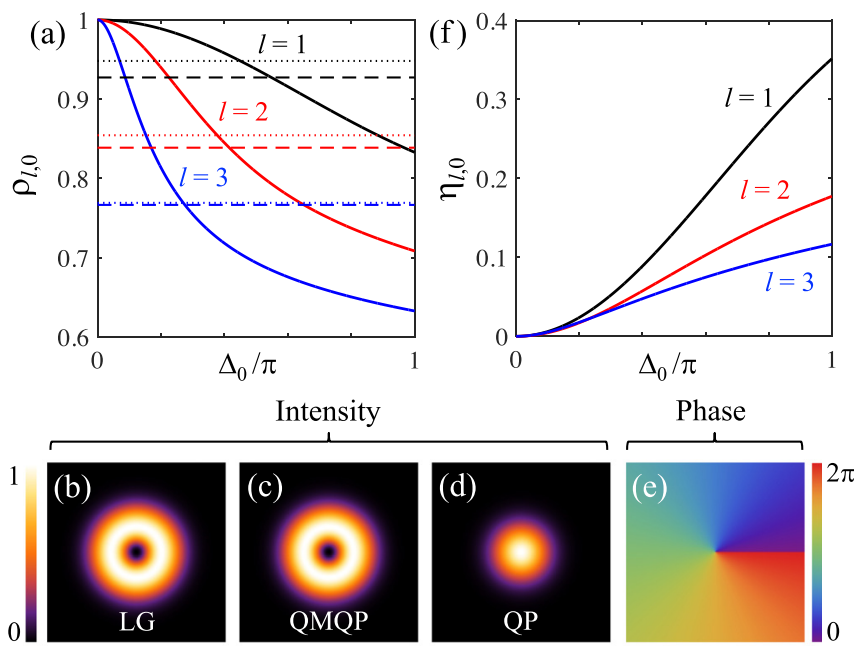

FIG. 1. (a) Simulated modality performances of QMQPs with $l \quad(1,2,3)$ and $p \quad 0$ for $0<\Delta_{0}<\pi$ (solid curves). Dashed lines refer to $\rho_{l, 0}$ values for QPs and dotted lines to the fabricated QMQPs. (b) (d) Output transverse intensity distribution of the targeted LG mode, QMQP with $\Delta_{0} \quad \pi / 5$ and $\mathrm{QP}$, for $(l, p) \quad(1,0)$, which are all associated with the same helical phase pro file in the transverse plane (e). (f) Simulated efficiency parameter for the same QMQPs as in panel (a). abovementioned improved features comes at the expense of a throughput decrease as $\Delta_{0}$ increases since only a fraction $\eta_{l, p}$ of the incident optical power is transformed into a contra-circularly polarized light field by the QMQPs, in contrast to QPs. This is gauged by evaluating the latter quantity whose expression is given by ${ }^{4}$

$$
\eta_{l, p}=\frac{4}{w_{0, \text { in }}^{2}} \int_{0}^{\infty} \sin ^{2}\left[\Delta_{l, p}(r) / 2\right] \exp \left(-2 r^{2} / w_{0, \text { in }}^{2}\right) r \mathrm{~d} r .
$$

The results are shown in Fig. 1(f) that points out a relatively modest efficiency up to $\eta_{l, 0} \simeq(0.15,0.06,0.03)$ in the range of $\Delta_{0}$ giving better modal performances than QPs for $l=(1,2,3)$, respectively. A practical tradeoff emerges from the earlier analysis between modality and efficiency regarding the experimental demonstration of QMQPs. The chosen target values are $\Delta_{0}=\pi / 5$ and $w_{0, \text { in }}=w_{0, \text { out }}=1 \mathrm{~mm}$, and the structured area corresponds to a disk of $5 \mathrm{~mm}$ in diameter.

Towards the realization of such QMQPs, one needs a technology enabling the combined fabrication of spacevariant retarders [i.e., $\Delta(r)$ ] and space-variant waveplates [i.e., $\psi(\phi)$ ]. Direct laser writing of self-assembled periodic subwavelength structures in the bulk of a UV fused silica glass slab by femtosecond laser pulses ${ }^{10,11}$ is chosen. Induced nanogratings have an orientation that is perpendicular to the linear polarization direction of the writing beam, exhibit optical anisotropy, and behave as negative uniaxial crystals. We used a femtoLAB micro-machining system (Altechna R\&D) equipped with $\mathrm{Yb}: \mathrm{KGW}$ femtosecond laser PHAROS (Light Conversion), placing the glass sample on a porous stone sample holder mounted on $\mathrm{XY}$ positioning stage (Aerotech) and using the following fabrication parameters: $1030 \mathrm{~nm}$ wavelength, 800 fs pulse duration, $200 \mathrm{kHz}$ repetition rate, and $2 \mathrm{~mm} / \mathrm{s}$ sample translation speed. The ultrafast laser radiation was focused via an aspheric lens with a numerical aperture $\mathrm{NA}=0.15$, at a distance $300 \mu \mathrm{m}$ below the surface of the glass sample.

Experimental demonstration is made for LaguerreGaussian modes with $(l ; p)=(1,2,3 ; 0)$. The radially varying retardance was ensured by adjusting the pulse energy during the fabrication, between $0.6 \mu \mathrm{J}$ (inner part), which roughly corresponds to the threshold value above which nanogratings suddenly appear, up to $1.2 \mu \mathrm{J}$ (outer part). The azimuthally varying optical axis orientation was controlled by translating the sample in the XY plane along an inward spiral trajectory with the synchronous rotation of polarization using a rotating half-wave plate. Images of the structures observed between crossed linear polarizers are shown in Fig. 2.

The qualitative visual inspection of the optical elements between crossed linear polarizers reveals on-axis deviations with respect to the designed structures, as shown in the bottom insets of Fig. 2. On one hand, the optical axis orientation pattern is twisted over an area of $\sim 100 \mu \mathrm{m}$ in radius possibly due to a lack of synchronization between position and polarization state of the writing pulsed beam at small radius. On the other hand, the visibility of the $2|l|$-brush intensity pattern does not vanish on-axis as expected from $\Delta \rightarrow 0$, which unveils unwanted retardation offset associated with the abrupt generation of nanogratings above a pulse energy threshold. 

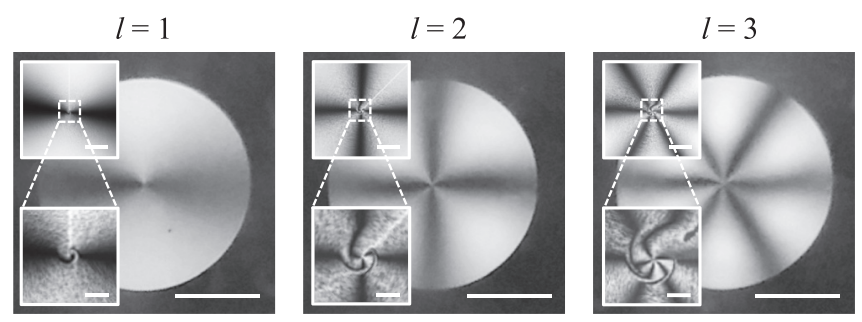

FIG. 2. Fabricated $5 \mathrm{~mm}$ diameter QMQPs with $l \quad(1,2,3)$ and $p \quad 0$ observed between crossed linear polarizers under natural white light illumi nation. Scale bars: $2 \mathrm{~mm}$ (main image), $500 \mu \mathrm{m}$ (top inset), and $100 \mu \mathrm{m}$ (bottom inset).

More quantitative insight is obtained by birefringent microoptical characterization using Abrio imaging system. This is summarized in Fig. 3 where the maps of optical axis orientation reveal a singular material structuring with topological charge $l / 2$ [panels (a) (c)] and birefringent phase retardation at $633 \mathrm{~nm}$ wavelength [panels (d) (f)] exhibit a good level of axisymmetry as expected by design. A retardance offset is systematically found as shown in Figs. 3(g) 3(i) where the measured azimuth average profiles $\langle\Delta\rangle(r)$ (dashed curves) are fitted to the function $\Delta_{\text {offset }}+\Delta_{0}\left(r / w_{0, \text { in }}\right)^{|l|}$ (see blue curves) that gives $\Delta_{\text {offset }}=0.726 \pm 0.004$ and $\Delta_{0}=0.976$ \pm 0.071 for the set of three structures. Although the fabricated structures differ from the ideal QMQP design, the modality of the realized optical elements [see the dotted line in Fig. 1(a)] is better than that of QP [see the dashed line in Fig. 1(a)].

The characterization of the generated shaped light beams is made by observing the intensity patterns at one Rayleigh distance $\left(z_{0}=\pi w_{0, \text { in }}^{2} / \lambda\right)$ from the sample and in the far field. This is done using the experimental setup shown in Figs. 4(a) and 5(a), respectively. The light source a continuous-wave Gaussian laser beam at a wavelength $\lambda=633 \mathrm{~nm}$ with $w_{0, \text { in }}$
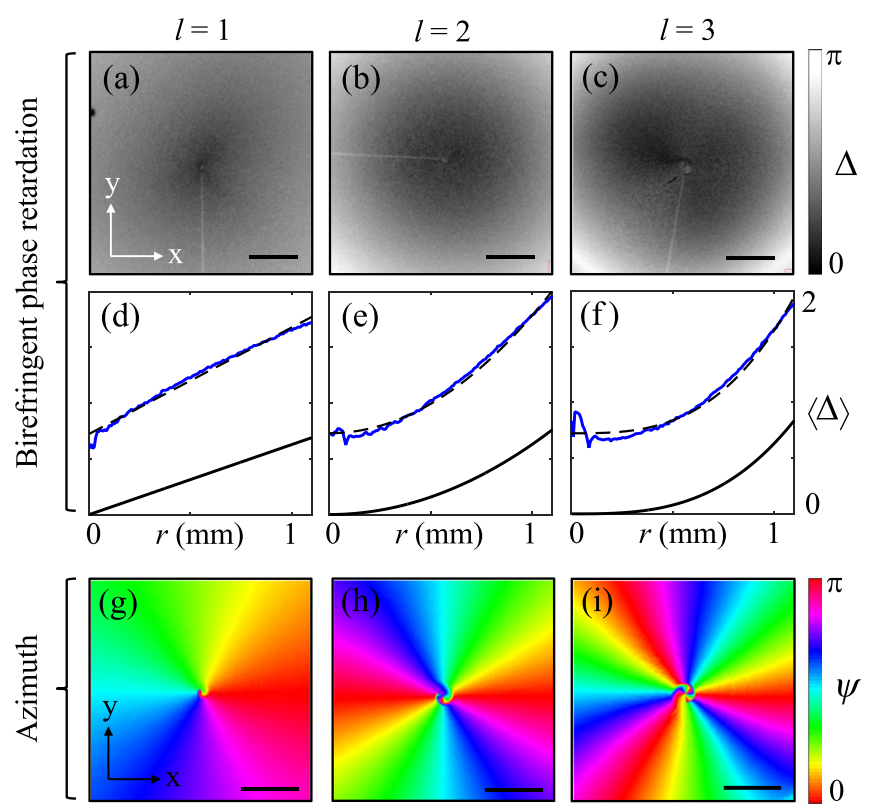

FIG. 3. (a) (c) Measured maps of birefringent phase retardation profiles at $633 \mathrm{~nm}$ wavelength for $l \quad(1,2,3)$ in the plane of the sample. Scale bar: $1 \mathrm{~mm}$. (d) (f) Measured azimuth average profiles (dashed curves), best fit (blue curves), and designed profile (solid curve). (g) (i) Measured maps of optical axis orientation. Scale bar: $1 \mathrm{~mm}$.

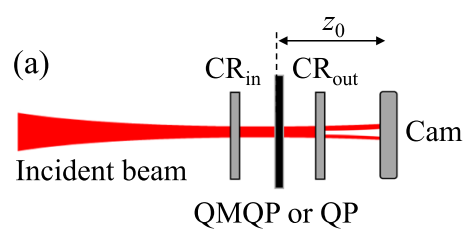

(b) Experiment

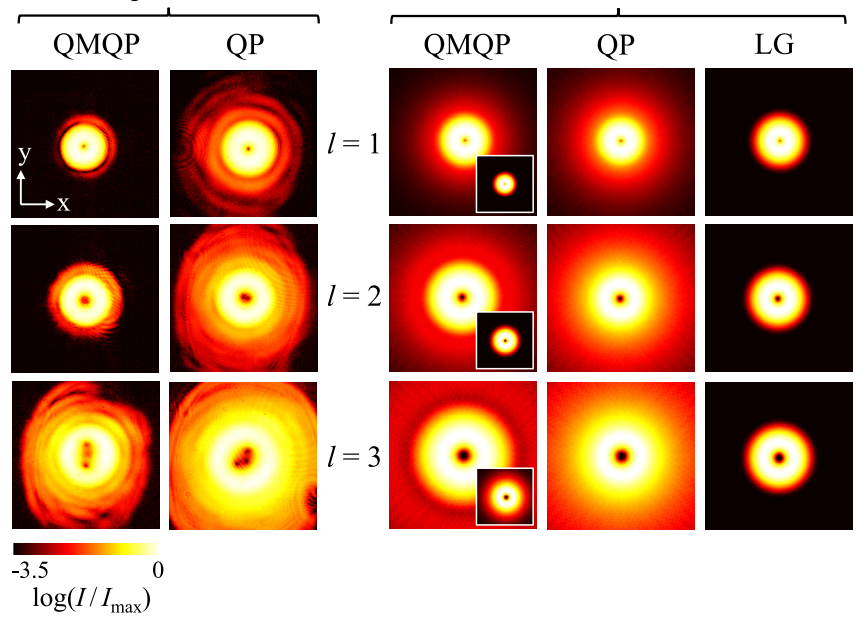

FIG. 4. (a) Experimental setup to study the shaped laser beam at one Rayleigh distance $z_{0}$ from either a QMQP or a QP illuminated at normal incidence with a circularly polarized Gaussian beam with wavelength $633 \mathrm{~nm}$ and beam waist $w_{0, \text { in }} 1 \mathrm{~mm}$ located in the plane of the QMQP that defines $z \quad 0$. The sample is placed between crossed circular polarizers $\left(\mathrm{CR}_{\text {in,out }}\right)$ and the transverse intensity pattern of the generated light field is directly observed with a camera (Cam). (b) Experimental data. (c) Simulations: QMQP main images refer to the fabricated structures and the inset refer to ideal QMQPs. $\mathrm{LG}_{l, 0}$ patterns are also shown for the sake of comparison. $I_{\max }$ is the maximal intensity of each image and the logarithmic intensity range refers to the 4096 levels of the 12 bit camera.

$=1 \mathrm{~mm}$, which gives $z_{0} \simeq 5 \mathrm{~m}$. The results at the finite distance are displayed in Fig. 4(b) that also provides a comparison with the case of QPs fabricated with the same femtosecond laser micro-machining technology. Characteristic doughnutshaped intensity patterns are obtained in both cases. Observed splitting of high-order optical phase singularities of order $|l|>$ 1 into $|l|$ unit charge singularities (which is here drastically emphasized by log scale representation) is mainly due to nonideal material structuring in the central part of the elements. Still, we note that even high-quality vortex generation methods lead to splitting of higher-order vortices that are very sensitive electromagnetic structures. ${ }^{12}$ This leads to deviation of the high-charge helical phase profile from the expected linear azimuthal dependence although the overall circulation of the phase around the center is fine. Also, the transverse spreading of the beam for QMQPs is less visible than in the case of QPs. This agrees with the simulated behavior shown in Fig. 4(c) where the intensity patterns are evaluated from twodimensional fast Fourier and inverse fast Fourier transforms $\left(\mathcal{F}\right.$ and $\left.\mathcal{F}^{1}\right)$ according to $I \propto\left|\mathcal{F}{ }^{1}\left[e^{i 2 \pi z_{0} / \lambda} \mathcal{F}\left(E_{\text {out }}^{\left({ }^{\sigma}\right)}\right)\right]\right|^{2}$. Note that the QMQP simulations are made taking into account the experimentally measured retardance profiles [Figs. 3(d) 3(f)] though the case of ideal design given by Eq. (2) is also shown in inset. On the other hand, the far-field intensity patterns are collected by placing a lens (L) in $f f$ configuration with respect to the optical element and the camera [see Fig. 5(a)]. The results are shown in Fig. 5(b) and compared to simulations in 


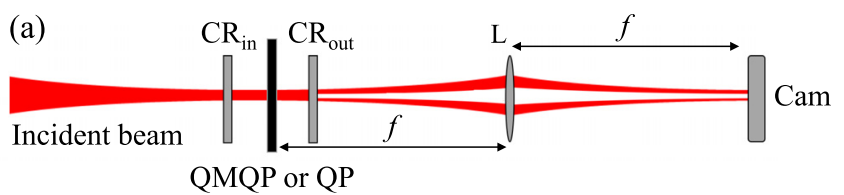

(b)

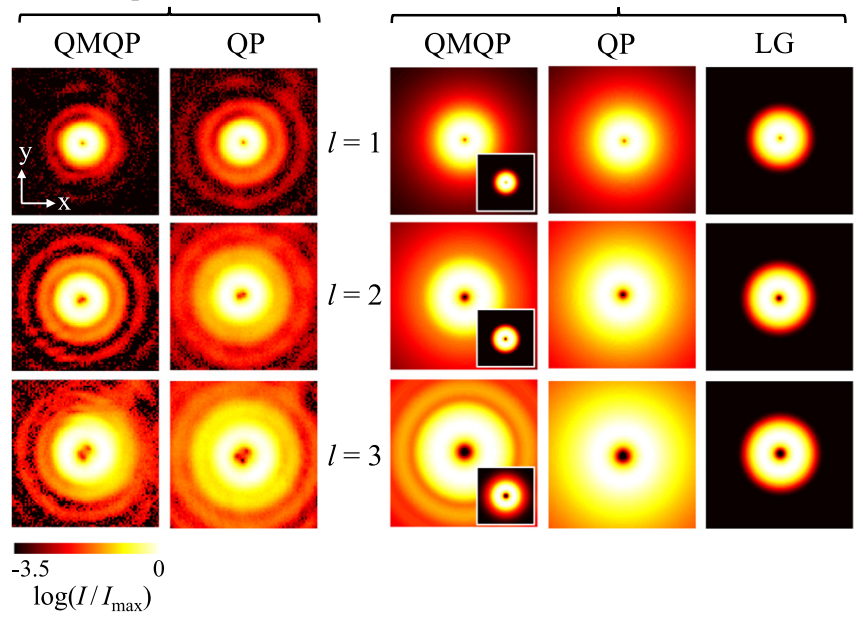

FIG. 5. (a) Experimental setup to study the shaped laser beam in the far field. It corresponds to the arrangement described in Fig. 4(a) where a spher ical lens with focal length $f \quad 300 \mathrm{~mm}$ is placed in $f f$ configuration between the optical element and the camera. (b) Experimental data. (c) Simulations: QMQP main images refer to the fabricated structures, and the inset refers to ideal QMQPs. $\mathrm{LG}_{l, 0}$ patterns are also shown for the sake of comparison. $I_{\max }$ is the maximal intensity of each image, and the logarithmic intensity range refers to the 4096 levels of the 12 bit camera.

Fig. 5(c) by using the Fourier relationship $I \propto\left|\mathcal{F}\left(E_{\text {out }}^{(\sigma)}\right)\right|^{2}$. Here again, the transverse beam spreading qualitatively indicates that the QMQP, even if nonideal, improves the modality of the LG beam shaping. Nevertheless, there is room for improvement, especially regarding the ability to get rid of the unwanted retardance offset of present technological approach. In particular, it would be interesting to consider the use of liquid crystal technology, which is mostly utilized for demonstrating q-plates. However, although arbitrary in-plane distribution of the optical axis orientation can be achieved at fixed retardance, ${ }^{13,14}$ it appears rather challenging to have the independent local control of both the birefringent phase retardation and the in-plane liquid crystal director orientation.

Summarizing, we proposed a quasi-modal approach to the concept of modal q-plates by exploring the limit of small birefringent phase retardation. Femtosecond direct laser writing of form birefringent structures in silica glass that combine space-variant properties of both the retardance and the optical axis orientation has been used to realize quasi-modal q-plates. Noteworthy the present approach preserves the spin-controlled management of topological charge of the generated optical vortex while keeping the modality unchanged, which is achieved at the expense of a reduced throughput of the modal optical element. Structural and optical characterization has been reported and we have pointed out the main technological challenges that should be addressed in order to realize better modal Laguerre-Gaussian beam shapers.

We acknowledge the financial support from French National Research Agency (ANR) in the frame of HYPERPHORB Project (ANR-15-CE30 0018).

${ }^{1}$ A. E. Siegman, Lasers (University Science Books, 1986).

${ }^{2}$ L. Allen, M. W. Beijersbergen, R. J. C. Spreeuw, and J. P. Woerdman, Phys. Rev. A 45, 8185 (1992).

${ }^{3}$ M. J. Padgett, Opt. Express 25, 11265 (2017).

${ }^{4}$ M. Rafayelyan and E. Brasselet, Opt. Lett. 42, 1966 (2017).

${ }^{5}$ G. Biener, A. Niv, V. Kleiner, and E. Hasman, Opt. Lett. 27, 1875 (2002).

${ }^{6}$ L. Marrucci, C. Manzo, and D. Paparo, Phys. Rev. Lett. 96, 163905 (2006).

${ }^{7}$ L. Marrucci, E. Karimi, S. Slussarenko, B. Piccirillo, E. Santamato, E. Nagali, and F. Sciarrino, J. Opt. 13, 064001 (2011).

${ }^{8}$ C. Schulze, S. Ngcobo, M. Duparré, and A. Forbes, Opt. Express 20, 27866 (2012).

${ }^{9}$ G. Vallone, Opt. Lett. 42, 1097 (2017).

${ }^{10}$ Y. Shimotsuma, P. G. Kazansky, J. Qiu, and K. Hirao, Phys. Rev. Lett. 91, 247405 (2003).

${ }^{11}$ M. Beresna, M. Gecevičius, P. G. Kazansky, and T. Gertus, Appl. Phys. Lett. 98, 201101 (2011).

${ }^{12}$ F. Ricci, W. Loffler, and M. P. van Exter, Opt. Express 20, 22961 (2012).

${ }^{13}$ J. Kim, Y. Li, M. N. Miskiewicz, C. Oh, M. W. Kudenov, and M. J. Escuti, Optica 2, 958 (2015).

${ }^{14}$ P. Chen, B. Y. Wei, W. Ji, S. J. Ge, W. Hu, F. Xu, V. Chigrinov, and Y. Q. Lu, Photonics Res. 3, 133 (2015). 